\title{
Asymptotic BER Analysis of Threshold Digital Relaying Schemes in Cooperative Wireless Systems
}

\author{
Furuzan Atay Onat, Yijia Fan, Halim Yanikomeroglu, and John S. Thompson
}

\begin{abstract}
Threshold relaying is an effective technique to achieve cooperative diversity in uncoded cooperative wireless networks, which suffer from error propagation due to detection errors at the relays. This paper analyzes the asymptotic end-toend (e2e) bit error rate (BER) of threshold digital relaying. A three node network with a source, destination and relay and with links experiencing independent Rayleigh fading is considered. It is shown that, as the average link signal-to-noise ratios (SNR) are increased simultaneously, the optimal threshold that minimizes the e2e BER increases as $\log (\mathrm{SNR})$. The resulting e2e BER decreases as $\log (\mathrm{SNR}) / \mathrm{SNR}^{2}$. Moreover, any threshold of the form $\log (c \mathrm{SNR})$, where $c$ is a positive constant, achieves the same order of e2e BER as the one achieved by the optimal threshold and provides dual diversity. A value of $c$ that performs very close to the optimal threshold is also proposed.
\end{abstract}

Index Terms-Cooperative diversity relaying, threshold maximum ratio combining, diversity order, selective digital relaying, SNR based selective relaying, multihop communication.

\section{INTRODUCTION}

C OOPERATIVE relaying can improve the e2e performance in wireless networks by creating independent paths from a source to its destination. The destination can combine signals from these independent paths using traditional combining methods such as Maximal Ratio Combining (MRC). Several relaying protocols to achieve cooperative diversity are proposed in [1]-[3]. According to the signal processing performed by the relay, cooperative relaying protocols can be classified into two categories: analog and digital. In analog relaying, the relay amplifies the noisy signal without detection. In digital relaying, the relay first detects and then remodulates the signal. The focus of the present paper is digital relaying.

Consider a source-destination pair and a single digital relay. If the detection at the relay is error-free, the destination is

Manuscript received May 3, 2007; revised November 3, 2007; accepted December 20, 2007. The associate editor coordinating the review of this paper and approving it for publication was D. Dardari. A short version of this paper appeared in the proceedings of the IEEE Wireless Communications and Networking Conference (WCNC), Las Vegas, Nevada, USA, 2008. This work was supported by Wireless Tech. Labs, Nortel Networks. John Thompson acknowledges the support of the Scottish Funding Council for the Joint Research Institute with the Heriot-Watt University which is a part of the Edinburgh Research Partnership.

F. A. Onat and H. Yanikomeroglu are with the Broadband Communications and Wireless Systems (BCWS) Centre, Dept. of Systems and Computer Engineering, Carleton University, Ottawa, ON, Canada (e-mail: \{furuzan, halim\}@sce.carleton.ca).

Y. Fan is with the Department of Electrical Engineering, Princeton University, Princeton, NJ (e-mail: yijiafan@ princeton.edu).

J. S. Thompson is with the Institute for Digital Communications, Joint Research Institute for Signal and Image Processing, School of Engineering and Electronics, University of Edinburgh, Edinburgh, UK (e-mail: john.thompson@ed.ac.uk).

Digital Object Identifier 10.1109/TWC.2008.070537. provided with two diversity branches: one from the relay and the other from the source. By combining these branches, the error probability at the destination is reduced significantly. However, retransmitting erroneous symbols from the relay causes the post-combining SNR at the destination to be very small and a symbol error at the destination becomes very likely. This latter event is usually called error propagation. If the relay retransmits all the symbols regardless of the reliability of its detection, i.e., simple digital relaying, the e2e diversity order is only one [4]. Hence, error propagation limits the e2e performance of digital relaying.

There are different ways to mitigate error propagation. In systems with embedded error detection codes, the relay can transmit only if no errors are detected. In this case, the relay can also correct some of the symbol errors by decoding and re-encoding the received data block. However, some coding schemes such as LDPC and turbo coding increases both the relay processing and delay. Moreover, in heterogeneous networks including devices with a wide range of capabilities, it might be preferable to use relaying schemes that are transparent to coding. When error detection is either unavailable or inefficient, an alternate approach is to use the instantaneous SNR of the source-relay link as an indication of the reliability of the relay detection. If the source-relay SNR is larger than a threshold, the probability of an error at the relay is small and hence the relay retransmits the signal. Otherwise, the relay remains silent. These kind of schemes are called Threshold Digital Relaying (TDR) [5]-[8].

In [6] Herhold et al. proposed a TDR scheme, where the threshold is selected jointly with the power fraction used by the relay and the source. They determine these parameters through numerical optimization. The BER of TDR schemes in a network with multi-antenna relays using a similar threshold function was studied by Adinoyi and Yanikomeroglu in [5]. In [7] the optimal threshold functions that minimize e2e BER have been derived analytically. It is shown that if the threshold is selected properly, TDR can improve the BER performance significantly compared to the simple relaying, where the relay always forwards the received data. In [8] this analysis has been extended to different levels of link SNR knowledge at the relay. In [9] Ponnaluri and Wilson considered a system with two parallel relays and equal gain combining at the destination. They showed that a threshold of the form $c \mathrm{SNR}^{\epsilon / 2}$ achieves diversity order of $d=3-\epsilon$, where $\epsilon>0$. With this threshold, despite the asymptotic diversity gain, the e2e BER performance does not improve significantly in the practical ranges of link SNRs, especially for low $\epsilon$ values.

Wang et al. proposed a new digital relaying scheme, called Link Adaptive Relaying (LAR), that aims to reduce error 
propagation by adapting the relay transmit power to the link SNRs, rather than transmitting with full power all the time [10], [11]. This idea is a generalization of TDR if the relay can control its power continuously and reduces to TDR if the relay can only perform on-off power control. In [10], the authors show that with their proposed power control scheme continuous version of LAR achieves full diversity while the on-off version of LAR has no diversity gain. In the present paper we prove that this result is not due to the limitation of on-off power control, but due to the specific threshold used in [10]. An optimal choice of threshold still achieves diversity order 2 .

Another line of research for improving the e2e BER of digital cooperative relaying focused on the enhancements that can be applied at the destination [12], [13]. Chen and Laneman derived the maximum-likelihood (ML) receiver and a piecewise linear receiver approaching the performance of the ML, that consider the possibility of symbol errors at the sourcerelay link [12]. In particular, the destination receiver makes use of the average BER at each relay during the first hop, which is sent to the destination by the relay. The authors show that the diversity order achieved by these receivers in digital relaying systems is bounded by $(M+1) / 2 \leq d \leq M / 2+1$ for $M$ even and is equal to $d=(M+1) / 2$ for $M$ odd, where $M-1$ is the number of relays. They also prove that for a single relay network with non-coherent detection, the e2e BER of their system decreases as $\log (\mathrm{SNR}) / \mathrm{SNR}^{2}$. In [13], a novel combining scheme to be employed at the destination is proposed. This scheme, which is called Cooperative MRC (C-MRC), exploits the instantaneous BER at source-relay links. The C-MRC achieves full diversity in uncoded digital relaying systems. However, it requires the relays to send their instantaneous BERs to the destination.

The schemes proposed in [12] and [13] place the computing burden on the destination while keeping the relays relatively simple. TDR, however, simplifies the destination process by making the relay smarter. The destination performs MRC only (if the relay retransmits) and it does not require any additional channel state information. Unlike [13], TDR exploits the instantaneous source-relay SNR at the relay, where it is available without additional signaling, rather than at the destination.

In this paper, we study the asymptotic e2e BER of the TDR in relation to the optimal threshold. After describing the system model in Section II, we summarize the prior results on the optimal threshold and the performance of the TDR in Section III. Section IV is dedicated to the analysis of the asymptotic BER of the optimal TDR in the high SNR regime. In this section we prove that the e2e BER of the optimal TDR decreases as $\log ($ SNR $) /$ SNR $^{2}$. Hence, the diversity order of the optimal TDR is 2. Then, in Section V, we show that any threshold that increases as $\gamma_{t}=\log (c$ SNR $)$ can also achieve the same asymptotic performance as the optimal TDR. We also propose a value of $c$ that results in a BER very close to the BER of the optimal threshold. In Section VI, we verify our results and compare the performance of the optimal TDR to several similar schemes proposed in the literature. Finally, Section VII summarizes the findings of the paper.

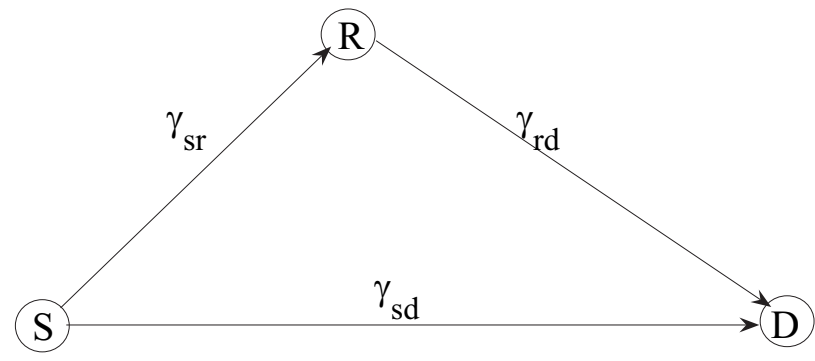

Fig. 1. Three node network.

\section{System Model}

This paper studies a simple network as shown in Fig 1, where a single relay $\mathrm{R}$ is used to assist the communication from a source node $\mathrm{S}$ to the destination node $\mathrm{D}$. A two phase digital relaying protocol is considered. In the first phase $S$ transmits a data block while $\mathrm{R}$ and $\mathrm{D}$ listens, and in the second phase $\mathrm{R}$ either retransmits the data or remains silent. If $\mathrm{R}$ chooses to remain silent, the second phase is idle. At the cost of increased complexity, $\mathrm{R}$ can send a short message to $\mathrm{S}$ about its decision and in the second phase $S$ can retransmit the same data. Since we assume slow fading as explained below, the retransmission by $\mathrm{S}$ does not provide any diversity gain. When $\mathrm{R}$ remains silent, the second phase can also be used by other nodes in the network to increase network throughput. However, in high SNR regime this gain is not significant either; as we will show in Section IV, in optimal TDR the probability that $\mathrm{R}$ remains silent goes to zero as SNR goes to infinity. That is, almost all the data blocks will be retransmitted by the relay.

Our system does not rely on any kind of coding and can process the data symbol by symbol as well. For simplicity, we assume that all the links use BPSK modulation. All the links experience independent Rayleigh fading. The instantaneous received SNR per bit for a link from node $i$ to node $j$ is denoted by $\gamma_{i j}$ and is given by $\gamma_{i j}=X_{i j}^{2} \sigma_{i j}^{2}$, where $X_{i j}^{2}$ is an exponential random variable with unit mean and $\sigma_{i j}^{2}$ is the average SNR. We represent the average SNR as $\sigma_{i j}^{2}=\lambda_{i j}$ SNR, where SNR is a reference signal-tonoise ratio, and $\lambda_{i j}$ is a scaling factor with respect to the reference SNR representing non-identical distance dependent loss and shadowing for different links. Link SNRs vary in time following independent block fading: $\gamma_{i j}$ is assumed to be constant for two blocks, precluding retransmit diversity, and a new independent realization is used for the next two blocks. The scalars $X_{i j}$, and thus $\gamma_{i j}$, are independent from link to link.

The instantaneous received SNR per bit for S-R, R-D, and S-D links are denoted by $\gamma_{s r}, \gamma_{r d}$, and $\gamma_{s d}$, respectively. Their average values are denoted by $\sigma_{s r}^{2}, \sigma_{r d}^{2}$, and $\sigma_{s d}^{2}$. An error event in the point-to-point link between node $i$ and node $j$ is represented by $\mathcal{E}_{i j}$. The error event that occurs after the destination combines the incorrectly regenerated relay signal and the source signal is referred to as error propagation and is denoted by $\mathcal{E}_{\text {prop. }}$. We use the term cooperative error for the event that an error occurs after the destination combines the correctly regenerated relay signal and the source signal, and denote this event by $\mathcal{E}_{\text {coop }}$. 
We assume that $\mathrm{R}$ has $\left\{\gamma_{s r}, \sigma_{r d}^{2}, \sigma_{s d}^{2}\right\}$ available in order to make relaying decisions. The relaying decisions are made by comparing the instantaneous S-R SNR $\gamma_{s r}$ to a threshold $\gamma_{t}$, i.e., $\mathbf{R}$ transmits only when $\gamma_{s r}>\gamma_{t}$. There are several ways of calculating the threshold $\gamma_{t}$ considered in the literature. Our work focuses on the performance with the optimal threshold value that minimizes e2e BER, which has been derived in [8]. We denote this threshold by $\gamma_{t}^{*}$ and we refer to the TDR using $\gamma_{t}^{*}$ as the optimal TDR. We note that the optimal threshold can also be calculated for the case where the relay has the instantaneous R-D and S-D SNRs, $\gamma_{r d}, \gamma_{s d}$. This additional information can improve e2e BER. However, the diversity order cannot be improved any further as the current model achieves full diversity.

\section{Performance of TDR And the Optimal THRESHOLD}

In this section, for completeness, we summarize the results from [8] on the optimal threshold and its BER performance for the model described in Section II.

\section{A. The e2e BER of TDR as a Function of $\gamma_{t}$}

The average e2e BER for a TDR protocol using threshold $\gamma_{t}$ can be expressed using the law of total probability:

$$
\begin{aligned}
\operatorname{BER}_{e 2 e}^{T D R}\left(\gamma_{t}\right) & =\mathbb{P}\left\{\gamma_{s r}>\gamma_{t}\right\}\left[\mathbb{P}\left\{\mathcal{E}_{s r} \mid \gamma_{s r}>\gamma_{t}\right\} \mathbb{P}\left\{\mathcal{E}_{\text {prop }}\right\}\right. \\
& \left.+\left(1-\mathbb{P}\left\{\mathcal{E}_{s r} \mid \gamma_{s r}>\gamma_{t}\right\}\right) \mathbb{P}\left\{\mathcal{E}_{\text {coop }}\right\}\right] \\
& +\mathbb{P}\left\{\gamma_{s r} \leq \gamma_{t}\right\} \mathbb{P}\left\{\mathcal{E}_{s d}\right\}
\end{aligned}
$$

Next, we derive the terms in (1) as functions of average link SNRs. If the SNR of the S-R link $\gamma_{s r}$ is below $\gamma_{t}$, the protocol falls back to direct transmission and has error probability equal to [14]

$$
\mathbb{P}\left\{\mathcal{E}_{s d}\right\}=\frac{1}{2}\left(1-\sqrt{\frac{\sigma_{s d}^{2}}{1+\sigma_{s d}^{2}}}\right) .
$$

Since $\gamma_{s r}$ is an exponential random variable with mean $\sigma_{s r}^{2}=$ $\lambda_{s r} \mathrm{SNR}$, the probability that $\gamma_{s r} \leq \gamma_{t}$ is equal to

$$
\mathbb{P}\left\{\gamma_{s r} \leq \gamma_{t}\right\}=1-\exp \left(-\gamma_{t} / \sigma_{s r}^{2}\right) .
$$

If $\gamma_{s r}>\gamma_{t}$, the probability of bit error at the S-R link decreases, but it remains nonzero regardless of the value of $\gamma_{t}$. The probability of bit error at the S-R link given that $\gamma_{s r}>\gamma_{t}$ is equal to

$\mathbb{P}\left\{\mathcal{E}_{s r} \mid \gamma_{s r}>\gamma_{t}\right\}=\int \frac{1}{2} \operatorname{erfc}\left(\sqrt{\gamma_{s r}}\right) p_{\gamma_{s r} \mid \gamma_{s r}>\gamma_{t}}\left(\gamma_{s r}\right) \mathrm{d} \gamma_{s r}$,

where erfc denotes the complementary error function. The term $p_{\gamma_{s r} \mid \gamma_{s r}>\gamma_{t}}$ is the conditional pdf of $\gamma_{s r}$ and is given by

$$
p_{\gamma_{s r} \mid \gamma_{s r}>\gamma_{t}}\left(\gamma_{s r}\right)=\exp \left(\gamma_{t} / \sigma_{s r}^{2}\right) \frac{1}{\sigma_{s r}^{2}} \exp \left(-\gamma_{s r} / \sigma_{s r}^{2}\right)
$$

for $\gamma_{s r}>\gamma_{t}$. Hence, the term $\mathbb{P}\left\{\mathcal{E}_{s r} \mid \gamma_{s r}>\gamma_{t}\right\}$ can be represented by

$$
\begin{aligned}
\mathbb{P}\left\{\mathcal{E}_{s r} \mid \gamma_{s r}>\gamma_{t}\right\}= & \frac{\exp \left(\gamma_{t} / \sigma_{s r}^{2}\right)}{2 \sigma_{s r}^{2}} \int_{\gamma_{t}}^{\infty} \operatorname{erfc}\left(\sqrt{\gamma_{s r}}\right) \\
& \times \exp \left(-\gamma_{s r} / \sigma_{s r}^{2}\right) \mathrm{d} \gamma_{s r},
\end{aligned}
$$

which can be calculated using integration by parts [6]:

$$
\begin{aligned}
\mathbb{P}\left\{\mathcal{E}_{s r} \mid \gamma_{s r}>\gamma_{t}\right\}= & \frac{1}{2} \operatorname{erfc}\left(\sqrt{\gamma_{t}}\right)-\frac{1}{2} e^{\gamma_{t} / \sigma_{s r}^{2}} \sqrt{\frac{\sigma_{s r}^{2}}{1+\sigma_{s r}^{2}}} \\
& \times \operatorname{erfc}\left(\sqrt{\gamma_{t}\left(1+\frac{1}{\sigma_{s r}^{2}}\right)}\right)
\end{aligned}
$$

When $\gamma_{s r}>\gamma_{t}$ and there is no bit error on the S-R link, then the probability of bit error at D after MRC is equal to [14, pp. 846-847]

$\mathbb{P}\left\{\mathcal{E}_{\text {coop }}\right\}= \begin{cases}\frac{1}{2}\left(1-s_{r d}\right)^{2}\left(1+\frac{1}{2} s_{r d}\right), & \sigma_{r d}^{2}=\sigma_{s d}^{2} ; \\ \frac{1}{2}\left[1-\frac{\sigma_{s d}^{2} s_{s d}-\sigma_{r d}^{2} s_{r d}}{\sigma_{s d}^{2}-\sigma_{r d}^{2}}\right], & \sigma_{r d}^{2} \neq \sigma_{s d}^{2} .\end{cases}$

In (7) $s_{r d}$ and $s_{s d}$ are defined as

$$
s_{r d}=\sqrt{\frac{\sigma_{r d}^{2}}{1+\sigma_{r d}^{2}}} \text { and } s_{s d}=\sqrt{\frac{\sigma_{s d}^{2}}{1+\sigma_{s d}^{2}}} .
$$

The probability of error propagation approaches to $\frac{\lambda_{r d}}{\lambda_{s d}+\lambda_{r d}}$ as the link SNRs increases. (See Appendix A for the proof.) As observed in [8], this limit is also a very good approximation for $\mathbb{P}\left\{\mathcal{E}_{\text {prop }}\right\}$ in the practical ranges of $\sigma_{r d}^{2}, \sigma_{s d}^{2}$. Hence, we use

$$
\mathbb{P}\left\{\mathcal{E}_{\text {prop }}\right\} \approx \frac{\lambda_{r d}}{\lambda_{s d}+\lambda_{r d}}=\frac{\sigma_{r d}^{2}}{\sigma_{s d}^{2}+\sigma_{r d}^{2}}
$$

for all the calculations in the rest of this paper.

The average e2e BER for a given threshold value can be calculated analytically by substituting (2), (3), and (6)-(8) into equation (1).

\section{B. The Optimal Threshold $\gamma_{t}^{*}$}

The threshold value $\gamma_{t}^{*}$ that minimizes the average e2e BER given in (1) is derived in [8]:

$$
\gamma_{t}^{*}= \begin{cases}\left(\operatorname{erfc}^{-1}(2 \delta)\right)^{2}, & \delta<0.5 ; \\ 0, & \text { otherwise }\end{cases}
$$

where $\delta$ is equal to

$$
\delta=\frac{\mathbb{P}\left\{\mathcal{E}_{\text {sd }}\right\}-\mathbb{P}\left\{\mathcal{E}_{\text {coop }}\right\}}{\mathbb{P}\left\{\mathcal{E}_{\text {prop }}\right\}-\mathbb{P}\left\{\mathcal{E}_{\text {coop }}\right\}} .
$$

By substituting (2), (7), and (8) into (10), $\delta$ is obtained as

$$
\delta\left(\sigma_{r d}^{2}, \sigma_{s d}^{2}\right) \approx \frac{\frac{1}{2}\left(1-s_{s d}\right)-\frac{1}{2}\left[1-\frac{\sigma_{s d}^{2} s_{s d}-\sigma_{r d}^{2} s_{r d}}{\sigma_{s d}^{2}-\sigma_{r d}^{2}}\right]}{\frac{\sigma_{r d}^{2}}{\sigma_{r d}^{2}+\sigma_{s d}^{2}}-\frac{1}{2}\left[1-\frac{\sigma_{s d}^{2} s_{s d}-\sigma_{r d}^{2} s_{r d}}{\sigma_{s d}^{2}-\sigma_{r d}^{2}}\right]} .
$$

\section{Asymptotic Performance of Optimal TDR}

In order to study the high SNR behavior of e2e BER, following an approach similar to the one in [15], we fix the parameters $\lambda_{s r}, \lambda_{r d}$, and $\lambda_{s d}$, and analyze the e2e BER as 
SNR $\rightarrow \infty$. Diversity order is a useful measure in quantifying the diversity benefit of any scheme in high SNR regime [15]. We adopt the following definition of diversity order given in $[15]^{1}$ :

$$
d=-\lim _{\mathrm{SNR} \rightarrow \infty} \frac{\log (\mathrm{BER})}{\log (\mathrm{SNR})} .
$$

We also use the following asymptotic notation:

Definition 1: Let $f(x)$ and $g(x)$ be two positive functions defined on real numbers. We say $f(x)=O(g(x))$, if $\limsup _{x \rightarrow \infty} \frac{f(x)}{g(x)}<\infty$.

Definition 2: Two functions $f(x)$ and $g(x)$ are called asymptotically equivalent, $f(x) \sim g(x)$, if $\lim _{x \rightarrow \infty} \frac{f(x)}{g(x)}=1$.

In rest of this section, we first show that the optimal threshold function $\gamma_{t}^{*}$ given in (9) increases logarithmically with SNR (Lemma 1). Then, using this result, we prove that if the relay sets its threshold to $\gamma_{t}^{*}$, the probability that it remains silent decreases as $\log (\mathrm{SNR}) / \mathrm{SNR}$ and the probability that the relay has a detection error decreases at least as fast as $1 /$ SNR $^{2}$ (Lemmas 2 and 3). Finally, we use all these results to show that the asymptotic e2e BER of the optimal TDR decreases as $\log (\mathrm{SNR}) / \mathrm{SNR}^{2}$ and the optimal TDR achieves diversity order 2 (Proposition 1).

We note that the BER of TDR is greater by a factor of $\log (\mathrm{SNR})$ than the BER of a traditional diversity system, where BER decreases as $1 / \mathrm{SNR}^{2}$ at large SNR. Since the diversity order represents the relation of the BER and SNR up to an exponential factor, the diversity order of TDR is still 2. Hence, we conclude that it is possible to achieve maximum diversity order in a single relay network using threshold relaying with the optimal threshold selection.

\section{A. Asymptotic Behavior of $\gamma_{t}^{*}, \mathbb{P}\left\{\gamma_{s r} \leq \gamma_{t}^{*}\right\}$, and $\mathbb{P}\left\{\mathcal{E}_{s r} \mid \gamma_{s r}>\right.$} $\left.\gamma_{t}^{*}\right\}$

The results on the asymptotic behavior of $\gamma_{t}^{*}, \mathbb{P}\left\{\gamma_{s r} \leq\right.$ $\left.\gamma_{t}^{*}\right\}$, and $\mathbb{P}\left\{\mathcal{E}_{s r} \mid \gamma_{s r}>\gamma_{t}^{*}\right\}$ are given in Lemmas 1,2 , and 3, respectively. See the Appendix for the proofs.

Lemma 1 (Asymptotic behavior of $\gamma_{t}^{*}$ ): The optimal threshold $\gamma_{t}^{*}$, given in (9), is upper and lower-bounded by two $\log$ functions for sufficiently large SNR. That is, there exist $c_{1}, c_{2}>0$ such that

$c_{1} \log (\mathrm{SNR})<\gamma_{t}^{*}(\mathrm{SNR})<c_{2} \log (\mathrm{SNR})$, as $\mathrm{SNR} \rightarrow \infty$.

Lemma 2 (Asymptotic behavior of $\mathbb{P}\left\{\gamma_{s r} \leq \gamma_{t}^{*}\right\}$ ): For sufficiently large SNR, $\mathbb{P}\left\{\gamma_{s r} \leq \gamma_{t}^{*}\right\}$ can be upper and lower bounded as follows. There exists $c_{1}^{\prime}, c_{2}^{\prime}>0$ such that

$c_{1}^{\prime} \frac{\log (\mathrm{SNR})}{\mathrm{SNR}}<\mathbb{P}\left\{\gamma_{s r}<\gamma_{t}^{*}\right\}<c_{2}^{\prime} \frac{\log (\mathrm{SNR})}{\mathrm{SNR}}$, as SNR $\rightarrow \infty$.

Lemma 3 (Asymptotic behavior of $\mathbb{P}\left\{\mathcal{E}_{s r} \mid \gamma_{s r}>\gamma_{t}^{*}\right\}$ ): If the relay uses the optimal threshold $\gamma_{t}^{*}$, then $\mathbb{P}\left\{\mathcal{E}_{s r} \mid \gamma_{s r}>\gamma_{t}^{*}\right\}$ can be upper bounded as follows. There exists a $c>0$ such that

$$
\mathbb{P}\left\{\mathcal{E}_{s r} \mid \gamma_{s r}>\gamma_{t}^{*}\right\}<c \frac{1}{\mathrm{SNR}^{2}}, \text { as } \mathrm{SNR} \rightarrow \infty .
$$

${ }^{1}$ Throughout this paper all the logarithms are in the natural base.

\section{B. Asymptotic e2e BER and Diversity Order of the Optimal} TDR

Proposition 1: The e2e BER of TDR, given in (1), using the threshold $\gamma_{t}^{*}$ satisfies $\operatorname{BER}_{e 2 e}^{T D R}\left(\gamma_{t}^{*}\right)=$ $O\left(\log (\mathrm{SNR}) / \mathrm{SNR}^{2}\right)$ and hence achieves the maximum diversity order of 2 .

Proof: Let us denote the first term of (1) as $P_{1}$ and the second term as $P_{2}$.

$$
\begin{aligned}
P_{1}=\mathbb{P}\left\{\gamma_{s r}>\gamma_{t}^{*}\right\} & {\left[\mathbb{P}\left\{\mathcal{E}_{s r} \mid \gamma_{s r}>\gamma_{t}^{*}\right\} \mathbb{P}\left\{\mathcal{E}_{\text {prop }}\right\}+\mathbb{P}\left\{\mathcal{E}_{\text {coop }}\right\}\right.} \\
- & \left.\mathbb{P}\left\{\mathcal{E}_{s r} \mid \gamma_{s r}>\gamma_{t}^{*}\right\} \mathbb{P}\left\{\mathcal{E}_{\text {coop }}\right\}\right],
\end{aligned}
$$

$P_{2}=\mathbb{P}\left\{\gamma_{s r} \leq \gamma_{t}^{*}\right\} \mathbb{P}\left\{\mathcal{E}_{s d}\right\}$.

In $P_{1}$, the term $\mathbb{P}\left\{\gamma_{s r}>\gamma_{t}^{*}\right\}=\exp \left(-\gamma_{t} /\left(\lambda_{s r} \mathrm{SNR}\right)\right)$ goes to 1 as SNR $\rightarrow \infty$. Since $\mathbb{P}\left\{\mathcal{E}_{\text {prop }}\right\} \sim \lambda_{r d} /\left(\lambda_{s d}+\lambda_{r d}\right)$ (from (35) in Appendix A) and $\mathbb{P}\left\{\mathcal{E}_{\text {coop }}\right\} \sim \frac{3}{16 \lambda_{r d} \lambda_{s d}} \frac{1}{\mathrm{SNR}^{2}}$, the decay rate of $P_{1}$ is equal to the minimum of the rates of $\mathbb{P}\left\{\mathcal{E}_{s r} \mid \gamma_{s r}>\gamma_{t}^{*}\right\}$ and $\mathbb{P}\left\{\mathcal{E}_{\text {coop }}\right\}$. From Lemma 3, $\mathbb{P}\left\{\mathcal{E}_{s r} \mid \gamma_{s r}>\right.$ $\left.\gamma_{t}^{*}\right\}=O\left(1 /\right.$ SNR $\left.^{2}\right)$. Hence, $P_{1}=O\left(1 /\right.$ SNR $\left.^{2}\right)$.

Since $\mathbb{P}\left\{\mathcal{E}_{s d}\right\} \sim \frac{1}{4 \lambda_{s d}} \frac{1}{\text { SNR }}$ and $\mathbb{P}\left\{\gamma_{s r} \leq \gamma_{t}^{*}\right\}$ $=O(\log (\mathrm{SNR}) / \mathrm{SNR})$ (from Lemma 2$), P_{2}=$ $O\left(\log (\mathrm{SNR}) / \mathrm{SNR}^{2}\right)$. The e2e BER of the optimal TDR satisfies

$$
\begin{aligned}
\mathrm{BER}_{e 2 e}^{T D R}\left(\gamma_{t}^{*}\right) & =P_{1}+P_{2}=O\left(1 / \mathrm{SNR}^{2}\right)+O\left(\log (\mathrm{SNR}) / \mathrm{SNR}^{2}\right) \\
& =O\left(\log (\mathrm{SNR}) / \mathrm{SNR}^{2}\right) .
\end{aligned}
$$

Thus, $\operatorname{BER}_{e 2 e}^{T D R}\left(\gamma_{t}^{*}\right)$ is limited by the second term $P_{2}$, which corresponds to the event that the SNR of the S-R link is below threshold.

The diversity order of the optimal TDR is equal to

$$
\begin{aligned}
d^{T D R} & =-\lim _{\mathrm{SNR} \rightarrow \infty} \frac{\log \left(\log (\mathrm{SNR}) / \mathrm{SNR}^{2}\right)}{\log (\mathrm{SNR})} \\
& =-\lim _{\mathrm{SNR} \rightarrow \infty} \frac{\log (\log (\mathrm{SNR}))}{\log \left(\mathrm{SNR}^{2}\right)} \\
& +\lim _{\mathrm{SNR} \rightarrow \infty} \frac{\log \left(\mathrm{SNR}^{2}\right)}{\log (\mathrm{SNR})}=2,
\end{aligned}
$$

since the term $\frac{\log (\log (\mathrm{SNR}))}{\log (\mathrm{SNR})} \rightarrow 0$ as $\mathrm{SNR} \rightarrow \infty$.

We note that the non-selective cooperative relaying protocol of [12] also achieves BER of $\log (\mathrm{SNR}) / \mathrm{SNR}^{2}$ using a piecewise linear detector at the destination and assuming noncoherent demodulation at the relay and the destination.

\section{AN APPROXIMATION TO THE OPTIMAL THRESHOLD}

The result given in Lemma 1 suggests that the threshold must increase logarithmically with SNR. In this section, we first prove that thresholds of the form $\gamma_{t}=\log (c \mathrm{SNR})$, achieves full diversity. Then, we propose a value for the constant $c$ based on the derivations in Section IV.

Proposition 2: The e2e BER of TDR, given in (1), using a threshold $\gamma_{t}=\log (c$ SNR $)$ satisfies $\operatorname{BER}_{e 2 e}^{T D R}\left(\gamma_{t}\right)=$ $O\left(\log (\mathrm{SNR}) / \mathrm{SNR}^{2}\right)$ and achieves the maximum diversity order of 2 for any real constant $c>0$. 
Proof: Substituting $\gamma_{t}=c \log (\mathrm{SNR})$ and $\sigma_{s r}^{2}=\lambda_{s r}$ SNR into (3), we obtain

$$
\begin{aligned}
\mathbb{P}\left\{\gamma_{s r} \leq \gamma_{t}\right\} & =1-\exp \left(-(\log (c \mathrm{SNR})) / \lambda_{s r} \mathrm{SNR}\right) \\
& =1-\left(\frac{1}{c \mathrm{SNR}}\right)^{\frac{1}{\lambda_{s r} \mathrm{SNR}}} \sim \frac{1}{\lambda_{s r}} \frac{\log (\mathrm{SNR})}{\mathrm{SNR}},
\end{aligned}
$$

where the last part is obtained from (41) given in the Appendix. Hence, the second term in (1), $P_{2}=\mathbb{P}\left\{\gamma_{s r} \leq\right.$ $\left.\gamma_{t}\right\} \mathbb{P}\left\{\mathcal{E}_{s d}\right\}=O\left(\log (\mathrm{SNR}) / \mathrm{SNR}^{2}\right)$. As in the proof of Proposition 1 , the order of the first term $P_{1}$ is determined by the term $\mathbb{P}\left\{\mathcal{E}_{s r} \mid \gamma_{s r}>\gamma_{t}\right\}$, i.e., $O\left(P_{1}\right)=O\left(\mathbb{P}\left\{\mathcal{E}_{s r} \mid \gamma_{s r}>\gamma_{t}\right\}\right)$. From (43) in the Appendix, for any threshold we have

$\mathbb{P}\left\{\mathcal{E}_{s r} \mid \gamma_{s r}>\gamma_{t}\right\}<\frac{1}{2 \lambda_{s r} \operatorname{SNR}} \operatorname{erfc}\left(\sqrt{\gamma_{t}}\right)<\frac{1}{2 \lambda_{s r} \mathrm{SNR}} e^{-\gamma_{t}}$,

where the last inequality follows from the following wellknown upper bound for erfc $[16]^{2}$ :

$$
\operatorname{erfc}(z)<e^{-z^{2}}
$$

By substituting $\gamma_{t}=\log (c$ SNR $)$, we obtain

$$
\mathbb{P}\left\{\mathcal{E}_{s r} \mid \gamma_{s r}>\gamma_{t}\right\}<\frac{1}{2 \lambda_{s r} c} \frac{1}{\mathrm{SNR}^{2}} .
$$

Hence, $\mathbb{P}\left\{\mathcal{E}_{s r} \mid \gamma_{s r}>\gamma_{t}\right\}=O\left(1 / \mathrm{SNR}^{2}\right)$ and $P_{1}=$ $O\left(1 / \mathrm{SNR}^{2}\right)$. Then, $\mathrm{BER}_{e 2 e}^{T D R}(\log (c \mathrm{SNR}))=P_{1}+P_{2}=$ $O\left(\log (\mathrm{SNR}) / \mathrm{SNR}^{2}\right)$ and the diversity order of TDR with $\gamma_{t}=\log (c \mathrm{SNR})$ is equal to 2 .

In order to obtain an approximation for $\gamma_{t}^{*}$, which is denoted by $\gamma_{t}^{*, \text { approx }}$, we use the upper bound given in (18) and the asymptotic expression given in (36) in the Appendix as approximations for erfc and $\delta$, respectively:

$\operatorname{erfc}(z) \approx e^{-z^{2}} \quad$ and $\quad \delta \approx \frac{1}{4} \frac{1}{\operatorname{SNR}} \frac{\lambda_{r d} \lambda_{s d}}{\lambda_{r d}+\lambda_{s d}}=\frac{1}{4} \frac{\sigma_{r d}^{2} \sigma_{s d}^{2}}{\sigma_{r d}^{2}+\sigma_{s d}^{2}}$

Then, $\gamma_{t}^{*, \text { approx }}$ is given by

$\gamma_{t}^{*, \text { approx }}= \begin{cases}-\log \left(\frac{1}{2}\left(\frac{1}{\sigma_{r d}^{2}}+\frac{1}{\sigma_{s d}^{2}}\right)\right), & \frac{1}{2}\left(\frac{1}{\sigma_{r d}^{2}}+\frac{1}{\sigma_{s d}^{2}}\right)<1 ; \\ 0, & \text { otherwise. }\end{cases}$

We note that for sufficiently large SNR, the condition $\frac{1}{2}\left(\frac{1}{\sigma_{r d}^{2}}+\frac{1}{\sigma_{s d}^{2}}\right)<1$ is satisfied and $\gamma_{t}^{* \text {,approx }}$ is equal to

$$
\begin{aligned}
\gamma_{t}^{*, \text { approx }} & =-\log \left(\frac{1}{2}\left(\frac{1}{\sigma_{r d}^{2}}+\frac{1}{\sigma_{s d}^{2}}\right)\right) \\
& =\log \left(2\left(\frac{1}{\lambda_{r d}}+\frac{1}{\lambda_{s d}}\right)^{-1} \mathrm{SNR}\right) .
\end{aligned}
$$

We observe that $\gamma_{t}^{* \text {,approx }}=\log (c \mathrm{SNR})$, where $c=$ $2\left(\frac{1}{\lambda_{r d}}+\frac{1}{\lambda_{s d}}\right)^{-1}$ is greater than zero. Hence, invoking Proposition 2, we conclude that with $\gamma_{t}^{*, a p p r o x}$, $\operatorname{BER}_{e 2 e}^{T D R}\left(\gamma_{t}^{*, \text { approx }}\right)=O\left(\log (\mathrm{SNR}) / \mathrm{SNR}^{2}\right)$ and the diversity order is equal to 2 . In (20), we notice that $\gamma_{t}^{* \text {,approx }}$ is equal to the logarithm of the harmonic mean of $\sigma_{s d}^{2}$ and $\sigma_{r d}^{2}$.

\footnotetext{
${ }^{2}$ There are tighter upper bounds for erfc, but this bound is sufficient for our purpose.
}

\section{RESULTS}

In this section, we compare the average BER of the optimal TDR to several schemes which are described below. As the two schemes that are simpler than TDR, we consider direct transmission and simple digital relaying. In simple digital relaying the relay always retransmits in the second phase. The average BER of this scheme is equal to

$$
\mathrm{BER}_{\text {e2e }}^{\text {simple }}=\mathbb{P}\left\{\mathcal{E}_{\text {sr }}\right\} \mathbb{P}\left\{\mathcal{E}_{\text {prop }}\right\}+\left(1-\mathbb{P}\left\{\mathcal{E}_{\text {sr }}\right\}\right) \mathbb{P}\left\{\mathcal{E}_{\text {coop }}\right\},
$$

which can be calculated analytically by substituting (2), (7), and (8) into (21).

The genie-aided relaying and receive diversity schemes are also considered as performance upper bounds. The genie-aided relaying is based on the hypothetical assumption that the relay has perfect error detection for each symbol. Hence, in phase 2 the relay retransmits only those symbols received correctly in phase 1. The e2e BER of the genie-aided digital relaying is equal to

$$
\mathrm{BER}_{e 2 e}^{\text {genie }}=\mathbb{P}\left\{\mathcal{E}_{s r}\right\} \mathbb{P}\left\{\mathcal{E}_{s d}\right\}+\left(1-\mathbb{P}\left\{\mathcal{E}_{\text {sr }}\right\}\right) \mathbb{P}\left\{\mathcal{E}_{\text {coop }}\right\},
$$

which can be calculated by substituting (2) and (7) into (22). The second upper bound, receive diversity, is obtained by assuming that the $\mathrm{S}-\mathrm{R}$ link is error-free, i.e., $\mathrm{BER}_{e 2 e}^{R x 2}=$ $\mathbb{P}\left\{\mathcal{E}_{\text {coop }}\right\}$, where $\mathbb{P}\left\{\mathcal{E}_{\text {coop }}\right\}$ is given in (7).

Finally, we compare the BER of the optimal TDR to other similar schemes proposed in the literature. The Link Adaptive Relaying (LAR) proposed in [10] aims to reduce error propagation by adapting the relay transmit power to the link SNRs rather than transmitting with full power $P_{\text {full }}$ all the time. If $\mathrm{R}$ is able to adapt its transmit power continuously, [10] proposes a scheme where $\mathrm{R}$ transmits with $\alpha \times P_{\text {full }}$, where the scaling factor $\alpha=\frac{\min \left(\gamma_{s r}, \sigma_{r d}^{2}\right)}{\sigma_{r d}^{2}}$. We call this scheme as LAR-cont. If $\mathrm{R}$ can perform only on-off power adaptation, then it can be seen that LAR simplifies to TDR with threshold function $\gamma_{t, L A R}=\sigma_{r d}^{2}$. We call this scheme as LAR-on/off. For all threshold based relaying schemes, the e2e BER can be calculated analytically by plugging in the appropriate threshold value as $\gamma_{t}$ in (1). We resort to Monte Carlo simulations to obtain the e2e BER of LAR-cont only.

In Fig. 2 we plot the average BER as a function of SNR in a symmetric network where $\lambda_{s r}=\lambda_{s d}=\lambda_{r d}=0 \mathrm{~dB}$. The on-off version of LAR has poor performance; its BER is larger than even the BER of the simple relaying. Since $\gamma_{t, L A R}$ increases linearly with SNR, from (40) we observe that $\mathbb{P}\left\{\gamma_{s r} \leq \gamma_{t, L A R}\right\}$ will be a constant independent of SNR. Then, in the e2e BER expression given in (1) the second term, $\mathbb{P}\left\{\gamma_{s r} \leq \gamma_{t, L A R}\right\} \times \mathbb{P}\left\{\mathcal{E}_{s d}\right\}$, decreases only as fast as the BER of the S-D link. Hence, the diversity order of on-off LAR is 1 , which has also been reported in [10]. This argument applies to any TDR scheme that uses a threshold increasing linearly with the SNR. The continuous version of LAR, which is shown to achieve full diversity in [10], performs better than all TDR schemes including the optimal TDR. However, the gap with the optimal TDR is very small. Fig. 3 shows the BER of all the schemes for an asymmetric network, where the direct link is weaker than the S-R and R-D links, a typical scenario where R is located on the line segment between S and D. Relative link 


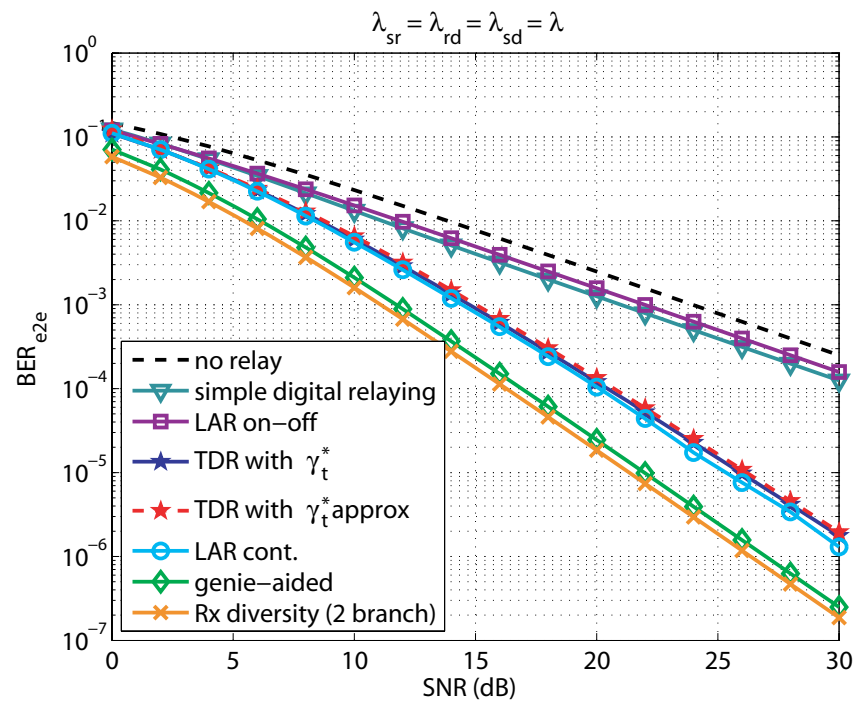

Fig. 2. The e2e BERs for different schemes as a function of SNR in a symmetric network, where $\lambda_{s r}=\lambda_{r d}=\lambda_{s d}=0 \mathrm{~dB}$.

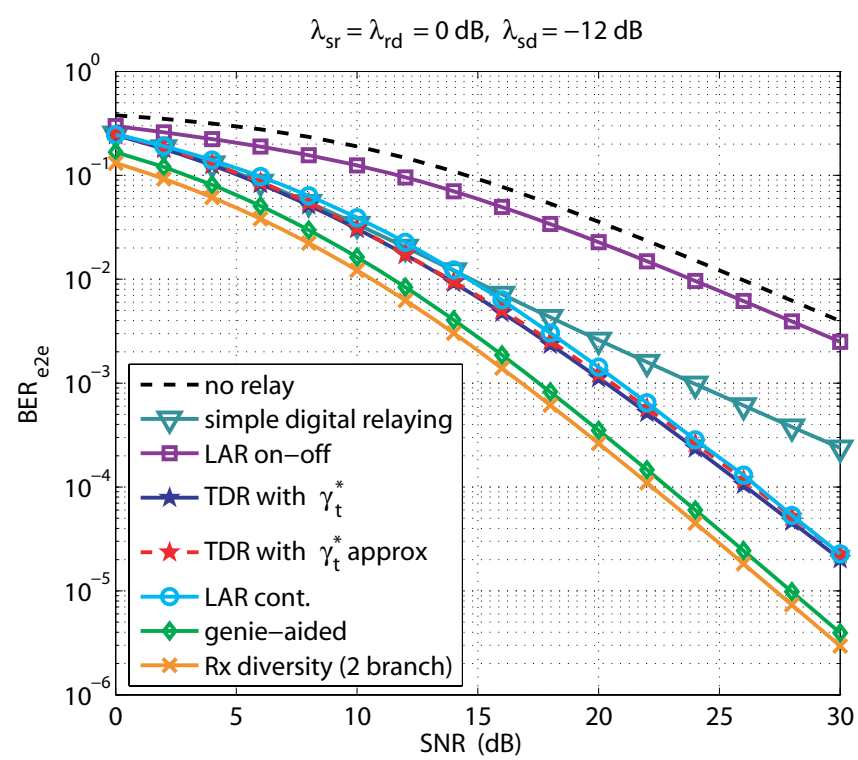

Fig. 3. The threshold values and e2e BERs for different schemes as a function of SNR in a nonsymmetric network, where $\lambda_{s r}=\lambda_{r d}=0 \mathrm{~dB}$ and $\lambda_{s d}=-12 \mathrm{~dB}$.

SNRs are selected as $\lambda_{s r}=\lambda_{r d}=0 \mathrm{~dB}$ and $\lambda_{s d}=-12 \mathrm{~dB}$. Unlike the symmetric case, here the optimal TDR outperforms continuous LAR by a very small margin. We note that the $\alpha$ value used in [10] for the continuous LAR is not optimized. Otherwise, it would always outperform the optimal TDR.

Fig. 4 and Fig. 5 show the variation of the optimal threshold and its approximation as a function of SNR, for the two sets of $\lambda$ values used in Fig. 2 and Fig. 3. Although there is an offset between the optimal threshold and the approximation, the two curves are almost parallel to each other. As seen in Fig. 2 and Fig. 3, the BER of the approximate threshold $\gamma_{t}^{*, a p p r o x}$ is very close to the BER of the optimal threshold.

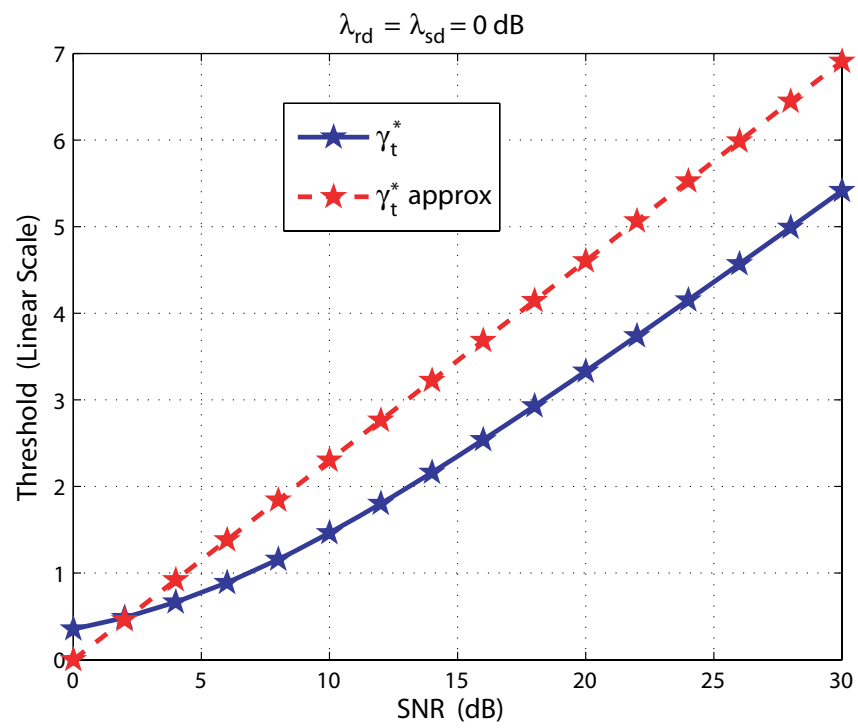

Fig. 4. The threshold values as a function of SNR in a with $\lambda_{r d}=\lambda_{s d}=0$ dB.

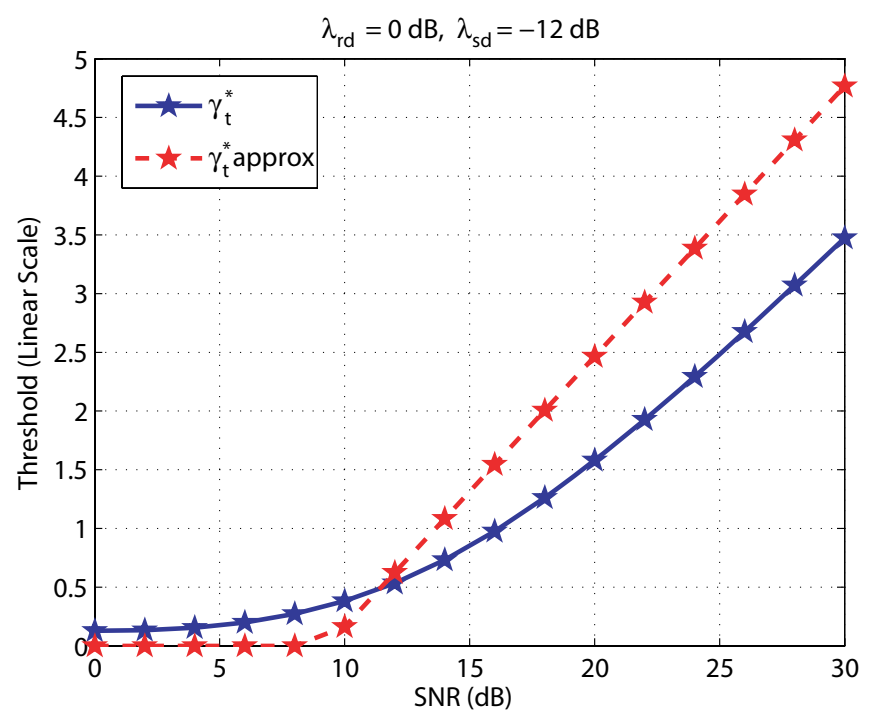

Fig. 5. The threshold values as a function of SNR in a nonsymmetric network, where $\lambda_{r d}=0 \mathrm{~dB}$ and $\lambda_{s d}=-12 \mathrm{~dB}$.

\section{CONCLUSIONS}

In this paper, we studied the asymptotic BER of threshold digital relaying in cooperative wireless networks. We showed that in a network with a single relay and independent Rayleigh fading links, in order to minimize e2e BER, the threshold used by the relay should increase logarithmically with the average link SNR. It is proven that this optimal threshold achieves dual diversity. We also showed that any threshold of the form $\log (c$ SNR $)$, where $c>0$ is a constant, achieves the same diversity order. Moreover, we derived a value for $c$ that results in a BER very close to the BER of the optimal TDR. The generalization of the problem to multiple relays remains as a future work topic. 


\section{APPENDIX}

A. Asymptotic Behavior of the Probability of Error Propagation

In this section we derive upper and lower bounds for $\mathbb{P}\left\{\mathcal{E}_{\text {prop }}\right\}$ and, invoking the Pinching Theorem, we prove that $\lim _{S N R \rightarrow \infty} \mathbb{P}\left\{\mathcal{E}_{\text {prop }}\right\}=\lambda_{r d} /\left(\lambda_{r d}+\lambda_{s d}\right)$. The probability of error propagation is given by $[8]$

$$
\begin{aligned}
\mathbb{P}\left\{\mathcal{E}_{\text {prop }}\right\} & =\frac{1}{2} \mathbb{E}_{\gamma_{r d}, \gamma_{s d}}\left[\operatorname{erfc}\left(g\left(\gamma_{s d}, \gamma_{r d}\right)\right)\right] \\
& =\frac{1}{2} \mathbb{E}_{\gamma_{r d}}\left[\mathbb{E}_{\gamma_{s d} \mid \gamma_{r d}}\left[\operatorname{erfc}\left(g\left(\gamma_{s d}, \gamma_{r d}\right)\right)\right]\right] \\
& =\frac{1}{2} \int_{0}^{\infty} \frac{1}{\sigma_{r d}^{2}} e^{-\gamma_{r d} / \sigma_{r d}^{2}} \\
& \times \int_{0}^{\infty} \operatorname{erfc}\left(g\left(\gamma_{s d}, \gamma_{r d}\right)\right) \frac{1}{\sigma_{s d}^{2}} e^{-\gamma_{s d} / \sigma_{s d}^{2}} \mathrm{~d} \gamma_{s d} \mathrm{~d} \gamma_{r d},
\end{aligned}
$$

where

$$
g\left(\gamma_{s d}, \gamma_{r d}\right)=\frac{\gamma_{s d}-\gamma_{r d}}{\sqrt{\gamma_{s d}+\gamma_{r d}}}
$$

Let us denote the upper and lower bounds for $\mathbb{P}\left\{\mathcal{E}_{\text {prop }}\right\}$, as $p_{l}$ and $p_{u}$, and the upper and lower bounds for the inner integral in (23), i.e. $\mathbb{E}_{\gamma_{s d} \mid \gamma_{r d}}\left[\operatorname{erfc}\left(g\left(\gamma_{s d}, \gamma_{r d}\right)\right)\right]$, as $f_{l}$ and $f_{u}$. Let $\mu_{g}$ and $\mu_{h}$ be the geometric and harmonic means of two positive numbers, where $\mu_{g}(x, y)=\sqrt{x y}$ and $\mu_{h}(x, y)=2 x y /(x+y)$. The square of $g\left(\gamma_{s d}, \gamma_{r d}\right)$ can be expressed as

$$
g^{2}\left(\gamma_{s d}, \gamma_{r d}\right)=\gamma_{s d}+\gamma_{r d}-2 \mu_{h}\left(\gamma_{s d}, \gamma_{r d}\right) .
$$

When $g\left(\gamma_{s d}, \gamma_{r d}\right) \geq 0$, i.e., $\gamma_{s d} \geq \gamma_{r d}$, making use of the fact that $\mu_{h}(x, y) \leq \mu_{g}(x, y)$ we reach the following inequality

$$
\begin{array}{r}
g^{2}\left(\gamma_{s d}, \gamma_{r d}\right) \geq \gamma_{s d}+\gamma_{r d}-2 \sqrt{\gamma_{s d} \gamma_{r d}}=\left(\sqrt{\gamma_{s d}}-\sqrt{\gamma_{r d}}\right)^{2} \\
\Rightarrow g\left(\gamma_{s d}, \gamma_{r d}\right) \geq \sqrt{\gamma_{s d}}-\sqrt{\gamma_{r d}}, \quad \gamma_{s d} \geq \gamma_{r d} .(24)
\end{array}
$$

For the region where $g\left(\gamma_{s d}, \gamma_{r d}\right)<0$, i.e., $\gamma_{s d}<\gamma_{r d}$, due to the fact that $m_{h}(x, y) \geq \min (x, y)$, we have

$$
\begin{gathered}
g^{2}\left(\gamma_{s d}, \gamma_{r d}\right) \leq \gamma_{s d}+\gamma_{r d}-2 \min \left(\gamma_{s d}, \gamma_{r d}\right) \Rightarrow \\
g\left(\gamma_{s d}, \gamma_{r d}\right) \geq-\sqrt{\gamma_{r d}-\gamma_{s d}}, \quad \gamma_{s d}<\gamma_{r d} .
\end{gathered}
$$

From (24) and (25), $\mathbb{E}_{\gamma_{s d} \mid \gamma_{r d}}\left[\operatorname{erfc}\left(g\left(\gamma_{s d}, \gamma_{r d}\right)\right)\right]$ can be bounded as follows:

$$
\begin{aligned}
& \mathbb{E}_{\gamma_{s d} \mid \gamma_{r d}}\left[\operatorname{erfc}\left(g\left(\gamma_{s d}, \gamma_{r d}\right)\right)\right] \\
= & \int_{0}^{\infty} \operatorname{erfc}\left(g\left(\gamma_{s d}, \gamma_{r d}\right)\right) \frac{1}{\sigma_{s d}^{2}} e^{-\gamma_{s d} / \sigma_{s d}^{2}} \mathrm{~d} \gamma_{s d} \leq f_{u}\left(\gamma_{r d}, \sigma_{s d}^{2}\right),
\end{aligned}
$$

where

$$
\begin{aligned}
f_{u}\left(\gamma_{r d}, \sigma_{s d}^{2}\right) & \triangleq \int_{0}^{\gamma_{r d}} \operatorname{erfc}\left(-\sqrt{\gamma_{r d}-\gamma_{s d}}\right) \frac{1}{\sigma_{s d}^{2}} e^{-\gamma_{s d} / \sigma_{s d}^{2}} \mathrm{~d} \gamma_{s d} \\
& +\int_{\gamma_{r d}}^{\infty} \operatorname{erfc}\left(\sqrt{\gamma_{s d}}-\sqrt{\gamma_{r d}}\right) \frac{1}{\sigma_{s d}^{2}} e^{-\gamma_{s d} / \sigma_{s d}^{2}} \mathrm{~d} \gamma_{s d}
\end{aligned}
$$

Following a similar approach, we also derive a lower bound for $\mathbb{E}_{\gamma_{s d} \mid \gamma_{r d}}\left[\operatorname{erfc}\left(g\left(\gamma_{s d}, \gamma_{r d}\right)\right)\right]$. It can be easily verified that

$$
\begin{array}{lll}
g\left(\gamma_{s d}, \gamma_{r d}\right) \leq \sqrt{\gamma_{s d}}-\sqrt{\gamma_{r d}}, & \gamma_{s d} \leq \gamma_{r d}, \quad \text { and } \\
g\left(\gamma_{s d}, \gamma_{r d}\right) \leq \sqrt{\gamma_{s d}-\gamma_{r d}}, & \gamma_{s d}>\gamma_{r d} .
\end{array}
$$

Thus, $f_{l}\left(\gamma_{r d}, \sigma_{s d}^{2}\right) \leq \mathbb{E}_{\gamma_{s d} \mid \gamma_{r d}}\left[\operatorname{erfc}\left(g\left(\gamma_{s d}, \gamma_{r d}\right)\right)\right]$, where

$$
\begin{aligned}
f_{l}\left(\gamma_{r d}, \sigma_{s d}^{2}\right) & \triangleq \int_{0}^{\gamma_{r d}} \operatorname{erfc}\left(\sqrt{\gamma_{s d}}-\sqrt{\gamma_{r d}}\right) \frac{1}{\sigma_{s d}^{2}} e^{-\gamma_{s d} / \sigma_{s d}^{2}} \mathrm{~d} \gamma_{s d} \\
& +\int_{\gamma_{r d}}^{\infty} \operatorname{erfc}\left(\sqrt{\gamma_{s d}-\gamma_{r d}}\right) \frac{1}{\sigma_{s d}^{2}} e^{-\gamma_{s d} / \sigma_{s d}^{2}} \mathrm{~d} \gamma_{s d}
\end{aligned}
$$

Next, we introduce two integrals, which we derive using integration by parts, to be used to calculate $f_{u}$ and $f_{l}$.

$$
\begin{aligned}
& I_{1}\left(a, b, c ; x_{0}, x_{1}\right)=\int_{x_{0}}^{x_{1}} \operatorname{erfc}(\sqrt{a x+b}) e^{-c x} \mathrm{~d} x \\
& =\left\{-\frac{1}{c} \operatorname{erfc}(\sqrt{a x+b}) e^{-c x}-\frac{1}{c} \sqrt{\frac{a}{a+c}}\right. \\
& \left.\left.\times(1-\operatorname{erfc}(\sqrt{(a+c) / a} \sqrt{a x+b})) e^{b c / a}\right\}\right]_{x_{0}}^{x_{1}},
\end{aligned}
$$

where $x_{0}, x_{1}, c \geq 0,(a+c) / a \geq 0$, and

$$
\begin{aligned}
& I_{2}\left(a, b, c ; x_{0}, x_{1}\right)=\int_{x_{0}}^{x_{1}} \operatorname{erfc}(a \sqrt{x}+b) e^{-c x} \mathrm{~d} x \\
& =\left\{-\frac{1}{c} \operatorname{erfc}(a \sqrt{x}+b) e^{-c x}-\frac{a}{c \sqrt{a^{2}+c}}\right. \\
& \times\left(1-\operatorname{erfc}\left(\sqrt{a^{2}+c} \sqrt{x}+a b / \sqrt{a^{2}+c}\right)\right) \\
& \left.\left.\times \exp \left(-b^{2}+a^{2} b^{2} /\left(a^{2}+c\right)\right)\right\}\right]_{x_{0}}^{x_{1}},
\end{aligned}
$$

where $x_{0}, x_{1}, c \geq 0$. Using $\operatorname{erfc}(-x)=2-\operatorname{erfc}(x),(26)$ is expressed in terms of $I_{1}$ and $I_{2}$. Then, by substituting (28) and (29) for $I_{1}$ and $I_{2}$, we obtain ${ }^{3}$

$$
\begin{aligned}
& f_{u}\left(\gamma_{r d}, \sigma_{s d}^{2}\right)=2\left(1-e^{-\gamma_{r d} / \sigma_{s d}^{2}}\right) \\
& -\left(1 / \sigma_{s d}^{2}\right) I_{1}\left(-1, \gamma_{r d}, 1 / \sigma_{s d}^{2}, 0, \gamma_{r d}\right) \\
& +\left(1 / \sigma_{s d}^{2}\right) I_{2}\left(1,-\sqrt{\gamma_{r d}}, 1 / \sigma_{s d}^{2}, \gamma_{r d}, \infty\right) \\
& =\operatorname{erfc}\left(-\sqrt{\gamma_{r d}}\right) \\
& -\frac{e^{-\gamma_{r d} / \sigma_{s d}^{2}}}{\sqrt{1-1 / \sigma_{s d}^{2}}}\left(1-\operatorname{erfc}\left(\sqrt{\gamma_{r d}} \sqrt{\left(1-1 / \sigma_{s d}^{2}\right)}\right)\right) \\
& -\frac{\exp \left(-\gamma_{r d} /\left(1+\sigma_{s d}^{2}\right)\right)}{\sqrt{1+1 / \sigma_{s d}^{2}}} \operatorname{erfc}\left(\sqrt{\gamma_{r d}} \frac{1 / \sigma_{s d}^{2}}{\sqrt{1+1 / \sigma_{s d}^{2}}}\right) .
\end{aligned}
$$

Similarly,

$$
\begin{aligned}
f_{l}\left(\gamma_{r d}, \sigma_{s d}^{2}\right) & =\frac{1}{\sqrt{1+1 / \sigma_{s d}^{2}}}\left[-e^{-\gamma_{r d} / \sigma_{s d}^{2}}-e^{-\gamma_{r d} /\left(1+\sigma_{s d}^{2}\right)}\right. \\
& \times\left(1-\operatorname{erfc}\left(\sqrt{\gamma_{r d}} /\left(\sigma_{s d}^{2} \sqrt{1+1 / \sigma_{s d}^{2}}\right)\right)\right) \\
& +\left(2-\operatorname{erfc}\left(\sqrt{\gamma_{r d}}\right)\right) \sqrt{1+1 / \sigma_{s d}^{2}} \\
- & \left.e^{-\gamma_{r d} /\left(1+\sigma_{s d}^{2}\right)}\left(1-\operatorname{erfc}\left(\sqrt{\gamma_{r d}} / \sqrt{1+1 / \sigma_{s d}^{2}}\right)\right)\right] .
\end{aligned}
$$

Upper and lower bounds for $\mathbb{P}\left\{\mathcal{E}_{\text {prop }}\right\}$ are found by using (23)

${ }^{3}$ It is assumed that $\sigma_{s d}^{2} \geq 1$, which is satisfied when SNR is sufficiently large. 
and taking the expectation of $f_{u}$ and $f_{l}$ w.r.t. $\gamma_{r d}$ :

$$
\begin{gathered}
f_{l}\left(\gamma_{r d}, \sigma_{s d}^{2}\right) \leq \mathbb{E}_{\gamma_{s d} \mid \gamma_{r d}}\left[\operatorname{erfc}\left(g\left(\gamma_{s d}, \gamma_{r d}\right)\right)\right] \leq f_{u}\left(\gamma_{r d}, \sigma_{s d}^{2}\right) \Rightarrow \\
\underbrace{\frac{1}{2} \mathbb{E}_{\gamma_{r d}}\left[f_{l}\left(\gamma_{r d}, \sigma_{s d}^{2}\right)\right]}_{p_{l}\left(\sigma_{r d}^{2}, \sigma_{s d}^{2}\right)} \leq \mathbb{P}\left[\mathcal{E}_{\text {prop }}\right] \leq \underbrace{\frac{1}{2} \mathbb{E}_{\gamma_{r d}}\left[f_{u}\left(\gamma_{r d}, \sigma_{s d}^{2}\right)\right]}_{p_{u}\left(\sigma_{r d}^{2}, \sigma_{s d}^{2}\right)} .
\end{gathered}
$$

Then we express $p_{l}$ and $p_{u}$ in terms of $I_{2}$ :

$$
\begin{aligned}
& p_{u}\left(\sigma_{r d}^{2}, \sigma_{s d}^{2}\right)= \\
& -\underbrace{\frac{\sigma_{s d}^{2}}{2\left(\sigma_{s d}^{2}+\sigma_{r d}^{2}\right)} \frac{1}{\sqrt{1-1 / \sigma_{s d}^{2}}}}_{t_{1}}+\underbrace{\frac{I_{2}\left(-1,0,1 / \sigma_{r d}^{2}, 0, \infty\right)}{2 \sigma_{r d}^{2}}}_{t_{2}} \\
& +\underbrace{\frac{I_{2}\left(\sqrt{1-1 / \sigma_{s d}^{2}}, 0,\left(\sigma_{r d}^{2}+\sigma_{s d}^{2}\right) /\left(\sigma_{r d}^{2} \sigma_{s d}^{2}\right), 0, \infty\right)}{2 \sigma_{r d}^{2} \sqrt{1-1 / \sigma_{s d}^{2}}}}_{t_{3}} \\
& -\underbrace{\frac{I_{2}\left(1 /\left(\sigma_{s d}^{2} \sqrt{1+1 / \sigma_{s d}^{2}}\right), 0, \frac{1+\sigma_{s d}^{2}+\sigma_{r d}^{2}}{\sigma_{r d}^{2}+\sigma_{r d}^{2} \sigma_{s d}^{2}}, 0, \infty\right)}{2 \sigma_{r d}^{2} \sqrt{1+1 / \sigma_{s d}^{2}}}}_{t_{4}} .
\end{aligned}
$$

We substitute $\sigma_{s d}^{2}=\lambda_{s d}$ SNR and $\sigma_{r d}^{2}=\lambda_{r d}$ SNR into (32), and take its limit as SNR $\rightarrow \infty$. Since $t_{1} \rightarrow(1 / 2) \lambda_{s d} /\left(\lambda_{s d}+\right.$ $\left.\lambda_{r d}\right), t_{2} \rightarrow 1, t_{3} \rightarrow 0$ and $t_{4} \rightarrow(1 / 2) \lambda_{s d} /\left(\lambda_{s d}+\lambda_{r d}\right)$, we conclude that

$$
\lim _{\mathrm{SNR} \rightarrow \infty} p_{u}=\frac{\lambda_{r d}}{\lambda_{r d}+\lambda_{s d}}
$$

Following the same approach for $p_{l}$, we obtain

$$
\begin{aligned}
& \begin{array}{l}
p_{l}\left(\gamma_{r d}, \sigma_{s d}^{2}\right)= \\
1-\underbrace{\frac{1}{2 \sqrt{1+1 / \sigma_{r d}^{2}}}\left(\frac{\sigma_{s d}^{2}}{\sigma_{s d}^{2}+\sigma_{r d}^{2}}+\frac{2\left(1+\sigma_{s d}^{2}\right)}{1+\sigma_{s d}^{2}+\sigma_{r d}^{2}}\right)}_{s_{1}} \\
+\underbrace{\frac{I_{2}\left(1 /\left(\sigma_{s d}^{2} \sqrt{1+1 / \sigma_{s d}^{2}}\right), 0, \frac{1+\sigma_{s d}^{2}+\sigma_{r d}^{2}}{\sigma_{r d}^{2}+\sigma_{r d}^{2} \sigma_{s d}^{2}}, 0, \infty\right)}{2 \sigma_{r d}^{2} \sqrt{1+1 / \sigma_{r d}^{2}}}}_{s_{3}} \\
+\underbrace{\frac{1}{2 \sigma_{r d}^{2} I_{2}\left(1,0,1 / \sigma_{r d}^{2}, 0, \infty\right)}}_{s_{s}} \\
\underbrace{I_{2}\left(1 / \sqrt{1+1 / \sigma_{s d}^{2}}, 0, \frac{1+\sigma_{s d}^{2}+\sigma_{r d}^{2}}{\sigma_{r d}^{2}+\sigma_{r d}^{2} \sigma_{s d}^{2}}, 0, \infty\right)}_{2 \sigma_{r d}^{2} \sqrt{1+1 / \sigma_{r d}^{2}}}
\end{array}
\end{aligned}
$$

Since $s_{1} \rightarrow(3 / 2) \lambda_{s d} /\left(\lambda_{s d}+\lambda_{r d}\right), s_{2} \rightarrow(1 / 2) \lambda_{s d} /\left(\lambda_{s d}+\right.$ $\left.\lambda_{r d}\right), s_{3} \rightarrow 0$, and $s_{4} \rightarrow 0$ as SNR $\rightarrow \infty$, we conclude that $\lim _{\mathrm{SNR} \rightarrow \infty} p_{l}=\frac{\lambda_{r d}}{\lambda_{r d}+\lambda_{s d}}$. Using this result combined with (33), the Pinching Theorem implies that

$$
\begin{aligned}
\lim _{\mathrm{SNR} \rightarrow \infty} p_{l} \leq \lim _{\mathrm{SNR} \rightarrow \infty} P\left\{\mathcal{E}_{\text {prop }}\right\} \leq \lim _{\mathrm{SNR} \rightarrow \infty} p_{u} \\
\Rightarrow \lim _{\mathrm{SNR} \rightarrow \infty} P\left\{\mathcal{E}_{\text {prop }}\right\}=\frac{\lambda_{r d}}{\lambda_{r d}+\lambda_{s d}} .
\end{aligned}
$$

\section{B. Proof of Lemma 1 - Asymptotic behavior of $\gamma_{t}^{*}$}

It can be easily verified that $\lim _{\mathrm{SNR} \rightarrow \infty} \frac{\delta(\mathrm{SNR})}{1 / \mathrm{SNR}}=$ $\frac{1}{4} \frac{\lambda_{s d}+\lambda_{r d}}{\lambda_{s d} \lambda_{r d}}$. Hence, $\delta(\mathrm{SNR})$ is asymptotically equivalent to

$$
\delta(\mathrm{SNR}) \sim \frac{1}{4} \frac{\lambda_{s d}+\lambda_{r d}}{\lambda_{s d} \lambda_{r d}} \frac{1}{\mathrm{SNR}} .
$$

For large SNR, $\delta(\mathrm{SNR})<1 / 2$. Thus, we ignore the second case in (9) and assume that $\gamma_{t}^{*}($ SNR $)=\left(\operatorname{erfc}^{-1}(2 \delta(\text { SNR }))\right)^{2}$.

We make use of the following inequality given in [17]:

$$
\sqrt{1-e^{-z^{2}}}<|\operatorname{erf}(z)|<\sqrt{1-e^{-2 z^{2}}} .
$$

By replacing $\operatorname{erfc}(z)=1-\operatorname{erf}(z)$, the threshold value is equal to $\gamma_{t}^{*}(\mathrm{SNR})=\left(\operatorname{erf}^{-1}(1-2 \delta(\mathrm{SNR}))\right)^{2}$. Since $\operatorname{erf}(z) \geq 0$ for all $z \geq 0$, and $\sqrt{\gamma_{t}^{*}} \geq 0$, we can write

$$
\sqrt{1-e^{-\gamma_{t}^{*}}}<\operatorname{erf}\left(\sqrt{\gamma_{t}^{*}}\right)<\sqrt{1-e^{-2 \gamma_{t}^{*}}} .
$$

Substituting $\operatorname{erf}\left(\sqrt{\gamma_{t}^{*}}\right)=1-2 \delta$ from (9), we obtain

$$
\frac{1}{2} \log \left(\frac{1}{4 \delta(1-\delta)}\right)<\gamma_{t}^{*}<\log \left(\frac{1}{4 \delta(1-\delta)}\right) .
$$

It can be easily verified that

$$
\lim _{\text {SNR } \rightarrow \infty} \log \left(\frac{1}{4 \delta(1-\delta)}\right) / \log (\text { SNR })=1 .
$$

Thus, there exists constants $b_{1}, b_{2}>0$ such that

$$
b_{1} \log (\mathrm{SNR})<\log \left(\frac{1}{4 \delta(1-\delta)}\right)<b_{2} \log (\mathrm{SNR}),
$$

and (13) holds for $c_{1}=b_{1} / 2$ and $c_{2}=b_{2}$, which concludes the proof.

\section{Proof of Lemma 2 - Asymptotic behavior of $\mathbb{P}\left\{\gamma_{s r} \leq \gamma_{t}^{*}\right\}$}

Since $\gamma_{s r}$ has mean $\sigma_{s r}^{2}=\lambda_{s r}$ SNR, the probability that $\gamma_{s r} \leq \gamma_{t}$, and hence relay remains silent, is equal to

$$
\begin{aligned}
\mathbb{P}\left\{\gamma_{s r} \leq \gamma_{t}\right\} & =1-\exp \left(-\frac{1}{\lambda_{s r}} \frac{\gamma_{t}(\mathrm{SNR})}{\mathrm{SNR}}\right) \\
& =1-\left(\exp \left(-\gamma_{t}(\mathrm{SNR})\right)\right)^{\frac{1}{\lambda_{s r} \mathrm{SNR}}}
\end{aligned}
$$

By substituting the bounds derived in Lemma 1 into (40), we obtain

$$
\begin{aligned}
& 1-\left(\exp \left(-c_{2} \log (\mathrm{SNR})\right)\right)^{\frac{1}{\lambda_{s r} \mathrm{SNR}}}<\mathbb{P}\left\{\gamma_{s r} \leq \gamma_{t}^{*}\right\} \\
& <1-\left(\exp \left(-c_{1} \log (\mathrm{SNR})\right)\right)^{\frac{1}{\lambda_{s r} \mathrm{SNR}}} \Rightarrow \\
& 1-\left(\frac{1}{\mathrm{SNR}}\right)^{\frac{c_{2}}{\lambda_{s r} \mathrm{SNR}}}<\mathbb{P}\left\{\gamma_{s r} \leq \gamma_{t}^{*}\right\}<1-\left(\frac{1}{\mathrm{SNR}}\right)^{\frac{c_{1}}{\lambda_{s r} \mathrm{SNR}}} .
\end{aligned}
$$

We note that

$$
\lim _{\mathrm{SNR} \rightarrow \infty} \frac{1-\left(\frac{1}{\mathrm{SNR}}\right)^{\frac{c}{\lambda_{s r} \mathrm{SNR}}}}{\log (\mathrm{SNR}) / \mathrm{SNR}}=\frac{c}{\lambda_{s r}}
$$

and hence,

$$
1-\left(\frac{1}{\mathrm{SNR}}\right)^{\frac{c}{\lambda_{s r} \mathrm{SNR}}} \sim \frac{c}{\lambda_{s r}} \frac{\log (\mathrm{SNR})}{\mathrm{SNR}} .
$$

Then, there exist positive constants $b_{1}^{\prime}$ and $b_{2}^{\prime}$ such that

$$
\frac{b_{1}^{\prime} c_{1}}{\lambda_{s r}} \frac{\log (\mathrm{SNR})}{\mathrm{SNR}}<\mathbb{P}\left\{\gamma_{s r} \leq \gamma_{t}^{*}\right\}<\frac{b_{2}^{\prime} c_{2}}{\lambda_{s r}} \frac{\log (\mathrm{SNR})}{\mathrm{SNR}}
$$


and (14) is satisfied for $c_{1}^{\prime}=\frac{b_{1}^{\prime} c_{1}}{\lambda_{s r}}$ and $c_{2}^{\prime}=\frac{b_{2}^{\prime} c_{2}}{\lambda_{s r}}$.

\section{Proof of Lemma 3 - Asymptotic behavior of $\mathbb{P}\left\{\mathcal{E}_{s r} \mid \gamma_{s r}>\right.$} $\left.\gamma_{t}^{*}\right\}$

Using Craig's formula given in [18], erfc can be represented as

$$
\operatorname{erfc}(z)=\frac{2}{\pi} \int_{0}^{\pi / 2} \exp \left(-z^{2} / \sin ^{2}(\theta)\right) \mathrm{d} \theta
$$

Substituting this alternate representation into (4) results in

$$
\begin{aligned}
& \mathbb{P}\left\{\mathcal{E}_{s r} \mid \gamma_{s r}>\gamma_{t}\right\} \\
& =\frac{e^{\gamma_{t} / \sigma_{s r}^{2}}}{2 \sigma_{s r}^{2}} \int_{\gamma_{t}}^{\infty} \operatorname{erfc}\left(\sqrt{\gamma_{s r}}\right) \exp \left(-\gamma_{s r} / \sigma_{s r}^{2}\right) \mathrm{d} \gamma_{s r} \\
& =\frac{e^{\gamma_{t} / \sigma_{s r}^{2}}}{\pi \sigma_{s r}^{2}} \int_{\gamma_{t}}^{\infty} \exp \left(-\frac{\gamma_{s r}}{\sigma_{s r}^{2}}\right) \int_{0}^{\pi / 2} \exp \left(-\frac{\gamma_{s r}}{\sin ^{2} \theta}\right) \mathrm{d} \theta \mathrm{d} \gamma_{s r} \\
& =\frac{e \gamma_{t} / \sigma_{s r}^{2}}{\pi \sigma_{s r}^{2}} \int_{0}^{\pi / 2} \int_{\gamma_{t}}^{\infty} \exp \left(-\gamma_{s r}\left(\frac{1}{\sin ^{2} \theta}+\frac{1}{\sigma_{s r}^{2}}\right)\right) \mathrm{d} \gamma_{s r} \mathrm{~d} \theta \\
& =\frac{1}{\pi} \int_{0}^{\pi / 2} \frac{\sin ^{2} \theta}{\sigma_{s r}^{2}+\sin ^{2} \theta} \exp \left(-\gamma_{t} / \sin ^{2} \theta\right) \mathrm{d} \theta .
\end{aligned}
$$

Since $\frac{\sin ^{2}(\theta)}{\sigma_{s r}^{2}+\sin ^{2}(\theta)}<\frac{1}{\sigma_{s r}^{2}}$ for any $\sigma_{s r}^{2}>0, \mathbb{P}\left\{\mathcal{E}_{s r} \mid \gamma_{s r}>\gamma_{t}\right\}$ is upper bounded by

$$
\begin{aligned}
\mathbb{P}\left\{\mathcal{E}_{s r} \mid \gamma_{s r}>\gamma_{t}\right\} & <\frac{1}{\pi} \int_{0}^{\pi / 2} \frac{1}{\sigma_{s r}^{2}} \exp \left(-\gamma_{t} / \sin ^{2} \theta\right) \mathrm{d} \theta \\
& =\frac{1}{2 \sigma_{s r}^{2}} \operatorname{erfc}\left(\sqrt{\gamma_{t}}\right)
\end{aligned}
$$

By substituting $\gamma_{t}=\gamma_{t}^{*}, \sigma_{s r}^{2}=\lambda_{s r} \mathrm{SNR}$, and $\operatorname{erfc}\left(\sqrt{\gamma_{t}^{*}}\right)=2 \delta$ from (9), (43) is simplified to

$$
\mathbb{P}\left\{\mathcal{E}_{s r} \mid \gamma_{s r}>\gamma_{t}^{*}\right\}<\frac{1}{\lambda_{s r}} \frac{\delta(\mathrm{SNR})}{\mathrm{SNR}} .
$$

Since $\delta(\mathrm{SNR}) \sim \frac{1}{4} \frac{\lambda_{s d}+\lambda_{r d}}{\lambda_{s d} \lambda_{r d}} \frac{1}{\operatorname{SNR}}$, there exists a constant $c^{\prime \prime}>$ 0 such that $\delta($ SNR $) \stackrel{\lambda_{s d} \lambda_{r d}}{<} c^{\prime \prime} /$ SNR for sufficiently large SNR. Hence, using (44) we obtain

$$
\mathbb{P}\left\{\mathcal{E}_{s r} \mid \gamma_{s r}>\gamma_{t}\right\}<\frac{1}{\lambda_{s r} \mathrm{SNR}} \delta(\mathrm{SNR})<\frac{c^{\prime \prime}}{\lambda_{s r}} \frac{1}{\mathrm{SNR}^{2}},
$$

and we conclude that (15) holds for $c=c^{\prime \prime} / \lambda_{s r}$.

\section{REFERENCES}

[1] N. Laneman, D. Tse, and G. Wornell, "Cooperative diversity in wireless networks: efficient protocols and outage behavior," IEEE Trans. Inform. Theory, vol. 50, no. 12, pp. 3062-3080, Dec. 2004.

[2] A. Sendonaris, E. Erkip, and B. Aazhang, "User cooperation diversitypart I: system description," IEEE Trans. Commun., vol. 51, no. 11, pp. 1927-1938, Nov. 2003.

[3] J. N. Laneman and G. W. Wornell, "Distributed space-time-coded protocols for exploiting cooperative diversity in wireless networks," IEEE Trans. Inform. Theory, vol. 49, no. 10, pp. 2415-2425, Oct. 2003.

[4] J. Boyer, D. D. Falconer, and H. Yanikomeroglu, "Multihop diversity in wireless relaying channels," IEEE Trans. Commun., vol. 52, no. 10, pp. 1820-1830, Oct. 2004.

[5] A. Adinoyi and H. Yanikomeroglu, "Cooperative relaying in multiantenna fixed relay networks," IEEE Trans. Wireless Commun., vol. 6, no. 2, pp. 533-544, Feb. 2007.

[6] P. Herhold, E. Zimmermann, and G. Fettweis, "A simple cooperative extension to wireless relaying," in Proc. International Zurich Seminar on Communications, 2004. [Online]. Available: http://wwwmns.ifn.et.tudresden.de/publications/2004/Herhold_P_IZS_04.pdf
[7] F. A. Onat, A. Adinoyi, Y. Fan, H. Yanikomeroglu, and J. S. Thompson, "Optimum threshold for SNR-based selective digital relaying schemes in cooperative wireless networks," in Proc. IEEE Wireless Communications and Networking Conference (WCNC), 2007.

[8] F. A. Onat, A. Adinoyi, Y. Fan, H. Yanikomeroglu, J. Thompson, and I. Marsland, "Threshold selection for SNR-based selective digital relaying in cooperative wireless networks," IEEE Trans. Wireless Commun., vol. 7, no. 11, pp. 4226-4237, Nov. 2008.

[9] S. P. Ponnaluri and S. G. Wilson, "On diversity of cooperative relaying protocols," in Proc. International Conference on Communications and Networking in China (ChinaCom), 2007.

[10] T. Wang, R. Wang, and G. Giannakis, "Smart regenerative relays for link-adaptive cooperative communications," in Proc. 40th Annual Conference on Information Sciences and Systems (CISS), Mar. 2006, pp. $1038-1043$.

[11] T. Wang, A. Cano, and G. Giannakis, "Link-adaptive cooperative communications without channel state information," in Proc. Military Communications Conference (MILCOM), Oct. 2006, pp. 1-7.

[12] D. Chen and J. N. Laneman, "Modulation and demodulation for cooperative diversity in wireless systems," IEEE Trans. Wireless Commun., vol. 5, no. 7, pp. 1785-1794, July 2006.

[13] T. Wang, A. Cano, G. B. Giannakis, and J. N. Laneman, "Highperformance cooperative demodulation with decode-and-forward relays," IEEE Trans. Commun., vol. 55, pp. 1427-1438, July 2007.

[14] J. G. Proakis, Digital Communications. McGraw-Hill, 2000.

[15] L. Zheng and D. Tse, "Diversity and multiplexing: a fundamental tradeoff in multiple antenna channels," IEEE Trans. Inform. Theory, vol. 49, no. 5, May 2003.

[16] J. M. Wozencraft and I. M. Jacobs, Principles of Communication Engineering. Waveland Press, 1990.

[17] D. V. Widder, Advanced Calculus. Eaglewood Cliffs, 1961, pp. 371.

[18] J. W. Craig, "A new, simple, and exact result for calculating the probability of error for two-dimensional signal constellations," in Proc. IEEE Military Communications Conference (MILCOM), Oct. 1991, pp. 571-575.

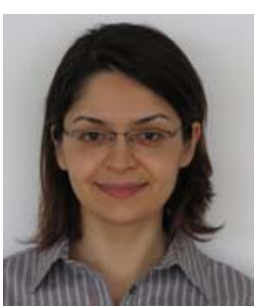

Furuzan Atay Onat received the B.S. degree in Electrical and Electronics Engineering from Middle East Technical University, Ankara, Turkey and the M.S degree in Electrical and Computer Engineering from Rutgers University, New Jersey, in 2000 and 2004, respectively. During the summer of 2006, she was an intern at Lucent Technologies, Bell Labs, NJ. Currently, she is a Ph.D. candidate in the department of Systems and Computer Engineering at Carleton University, Ottawa, Canada. Her research interests are in wireless communications and networking with special emphasis on multi-hop networks and cooperative communications.

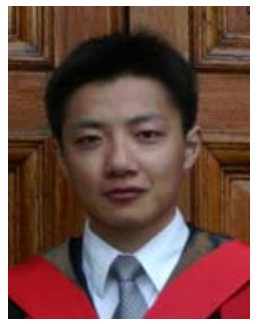

Yijia (Richard) Fan received his BEng degree in electrical engineering from Shanghai Jiao Tong University (SJTU), Shanghai, P.R. China, in July 2003, and $\mathrm{PhD}$ degree from the Institute for Digital Communications, University of Edinburgh, UK, March, 2007. His PhD project was fully funded by Engineering and Physical Sciences Research Council (EPSRC), UK. He is currently a postdoctoral research associate in Department of Electrical Engineering, Princeton University. His research interests include signal processing, information theory and their applications in future wireless networks. 


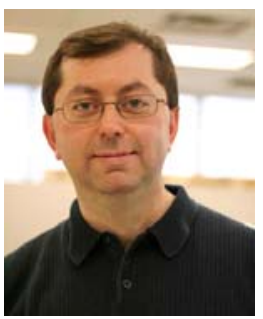

Halim Yanikomeroglu Halim Yanikomeroglu was born in Giresun, Turkey, in 1968. He received a B.Sc. degree in Electrical and Electronics Engineering from the Middle East Technical University, Ankara, Turkey, in 1990, and a M.A.Sc. degree in Electrical Engineering (now ECE) and a Ph.D. degree in Electrical and Computer Engineering from the University of Toronto, Canada, in 1992 and 1998, respectively. He was with the R\&D Group of Marconi Kominikasyon A.S., Ankara, Turkey, from January 1993 to July 1994.

Since 1998 Dr. Yanikomeroglu has been with the Department of Systems and Computer Engineering at Carleton University, Ottawa, where he is now an Associate Professor; he is also the Associate Chair for Graduate Studies in the department. Dr. Yanikomeroglu's research interests cover many aspects of the physical, medium access, and networking layers of wireless communications with a special emphasis on multihop/relay/mesh networks and cooperative communications.

Dr. Yanikomeroglu has been involved in the steering committees and technical program committees of numerous international conferences in communications; he has also given 15 tutorials in such conferences. He has been involved in the organization of the IEEE Wireless Communications and Networking Conference (WCNC) over the years. He is a member of the WCNC Steering Committee, and he was the Technical Program Co-Chair of WCNC 2004. He is the Technical Program Committee Chair of the IEEE WCNC 2008

Dr. Yanikomeroglu was an editor for IEEE TRANS ACTIONS ON WIRELESS COMmunications [2002-2005] and IEEE COMMUNICATIONS SuRveYs
\& TUTORIALS [2002-2003], and a guest editor for WILEY JOURNAL ON Wireless COMMUNicATIONS \& Mobile COMPuting. He was an Officer of the IEEE's Technical Committee on Personal Communications (Chair: 2005-06, Vice-Chair: 2003-04, Secretary: 2001-02), and he was also a member of the IEEE Communications Society's Technical Activities Council (200506). Dr. Yanikomeroglu is also a registered Professional Engineer in the province of Ontario, Canada.

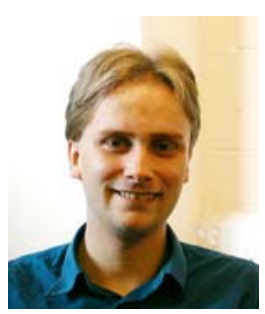

John S. Thompson received his BEng and $\mathrm{PhD}$ degrees from the University of Edinburgh in 1992 and 1996, respectively. From July 1995 to August 1999, he worked as a postdoctoral researcher at Edinburgh, funded by the UK Engineering and Physical Sciences Research Council (EPSRC) and Nortel Networks. Since September 1999, he has been a lecturer at the School of Engineering and Electronics at the University of Edinburgh. In October 2005, he was promoted to the position of reader. His research interests currently include signal processing algorithms for wireless systems, antenna array techniques and multihop wireless communications. He has published approximately 100 papers to date including a number of invited papers, book chapters and tutorial talks, as well as co-authoring an undergraduate textbook on digital signal processing. He is currently editor-in-chief of the IET Signal Processing journal and was recently a technical programme co-chair for the IEEE International Conference on Communications (ICC) 2007, held in Glasgow in June 2007. 